\title{
Application of Human-Machine Interactive Translation Model and Its Implications
}

\author{
Wang Zhaohui \\ Faculty of International Languages, Xiamen University of Technology \\ Xiamen, China
}

\begin{abstract}
Machine translation has developed from statistical machine translation to neural machine translation (also called AI translation). With the quality of AI translation being much superior to that of statistical machine translation, an epochmaking revolution has taken place in the field of translation technology. This paper discusses the application of AI translation in a human-machine interactive model, which has important enlightenment and practical significance for translation industry, translation teaching and research, as well as translation practice.
\end{abstract}

Keywords-human-machine interactive translation model; AI translation; application; implication

\section{INTRODUCTION}

At the FIT 20th World Congress, the combination of human translation and translation technology attracted much attention, highlighting a turning point in translation studies today - the technological turn [1]. The research on translation technology in China has reached its climax in the past decade, with the focus on basic and applied research on machine translation (MT) [2]. However, such research mainly focuses on the traditional machine translation, namely, statistical machine translation (SMT). There has been a lack of profound research on neural machine translation (NMT, hereinafter also referred to as AI translation) in the last two years, as well as a lack of innovative quality assessment of AI translation. Only some discussions on the quality of SMT were made [3-4].

In fact, with the rapid development of AI translation, it can be said that an epoch-making technological revolution is quietly emerging in the field of translation technology. This technological revolution has come suddenly and rapidly, but it has not been paid close attention to by translation researchers, and relevant studies are quite few.

The development of AI translation will bring great influence on and changes to translation industry, translation teaching and research, and translation practice. The interaction between machine translation and human translation, such as post-editing, however, has not been discussed much in China, with only a few papers ever published.

Under the background of the rise of translation technology in the big data era, both literary translation and non-literary translation can be done by AI translation. As a new translation

Funded by the Humanities and Social Science Foundation of the Ministry of Education of China (No. 17YJA740050). model, human-machine interactive translation is different from the traditional translation behavior in many aspects, which is worthy of in-depth study and discussion. This paper is an attempt to discuss the application of AI translation in a humanmachine interactive model and its implications.

\section{ABOUT AI TRANSLATION}

Machine translation (MT), according to the definition of ISO standard, is an automatic translation of a text or speech from one natural language to another using the computer system. It is different from computer-assisted translation (CAT), which is a translation mode in which human translation is assisted with the translation memory and term bank of CAT tools.

MT can be divided into two types according to its development stages: statistical machine translation (SMT), and neural machine translation (NMT), which began to thrive in 2017. Previous discussions on MT generally refer to SMT if it is not clearly identified as NMT or AI translation. However, in the past two years, almost all machine translation service, such as Google translation, Bing translation, Baidu translation, Sogou translation, Youdao translation and Xiaoniu translation, have shifted from SMT to NMT.

Traditionally, the evaluation of SMT is negative, and SMT is often considered to be full of mistakes and thus useless. For example, Wang Kefei, in his discussion about the quality of machine translation, pointed out that machine translation was unable to deal with complex Chinese expressions and had poor word order adjustment ability [3]. However, for present AI translation, new features have emerged and translation quality has greatly improved.

Taking the Google Neural Machine Translation (GNMT) system as an example, compared with the previous phrasebased machine translation system, GNMT is much better in translation quality. With the help of bilingual evaluators, Google researchers measured sample sentences from Wikipedia and news websites and found that GNMT reduced translation errors by more than $55 \%-85 \%$ in multiple major language pairs [5]. Here is an example of GNMT's superiority to Google's statistical machine translation in terms of translation quality. The original is a Chinese sentence about the Zurich office of Wegelin. The Google SMT (GSMT) and GNMT put it into English respectively: 
(a) GSMT: to provide confidential business 271 years of Wegelin Zurich Bank (the Zurich office of Wegelin) has always been not noticeable.

(b) GNMT: The Zurich office of Wegelin, which provides confidential business for 271 years, has always been inconspicuous.

It's obvious that GNMT is far better than Google SMT in terms of grammar and readability.

What's more, AI translation can sometimes even surpass human translation in some ways. Take translation of terminology as an example. Due to its large collection of bilingual terms, AI translation may do better than human translation when it comes to translating some terms. In an exercise done by junior English majors, some of the students put "Changchun yiqi" literally into "Changchun-based No. 1 Auto Plant", while quite a few AI translation engines, such as Google translation and Sougou translation, rendered it correctly into "Changchun FAW", which is its official name in English. The AI translation here is more accurate than human translation, and there are many examples of this kind. Therefore, the quality of machine translation is greatly improved, which is closely related to AI technology. It goes without saying that there are many terms for which the data banks of AI translation have no equivalent translations. On many occasions, human translation is required to come up with correct term translations for these terms.

AI translation has emerged in the field of non-literary translation, and the gap between AI translation and human translation is narrowing. In a human-machine interactive model, the draft translation can be done by machine translation, and the translator is just responsible for post-editing. As far as translation teaching, translation studies and translation practice are concerned, great importance should be attached to AI translation, especially to the application of the human-machine interactive translation model.

\section{HUMAN-MACHINE INTERACTIVE TRANSLATION MODEL}

According to $\mathrm{Hu}$ Kaibao, machine translation will take on more and more translation tasks, and will partly replace human translation, but will not completely replace human translation in the end. The future translation profession will be in an era of human-machine coexistence and human-machine complementarity [6]. Thus, the human-machine interactive translation model is the best choice at present, and its specific operation methods are suggested as follows:

Firstly, CAT pre-translation. CAT tools help enhance human translation efficiency and accuracy with their translation memories and term banks. It is suggested, therefore, that a translation project be handled with CAT's pre-translation, through which sentences matched with those of the translation memories will be translated automatically, thus saving a lot of human efforts. It may not be very helpful, however, if what to be translated is what has never been translated before. Thus the need for AI translation, which is fast and quite accurate as well.

Secondly, AI translation. It can also be carried out through CAT tools, many of which, such as Snowman CAT and Trados, provide AI translation through API or plugins. AI translation systems can automatically translate the unmatched sentences in the CAT memories. The translation speed is extremely fast, while the accuracy depends on the text types. Generally, the translation quality of non-literary subjects are better than that of literary ones.

Thirdly, human post-editing of AI translation. Due to the errors that are expected for AI translation, human efforts are needed to spot and correct errors through post-editing.

Through the above human-machine interactive model, the overall translation efficiency is much higher than that of the traditional human model, the traditional CAT model, and the AI translation model alone.

If there are a large number of similar texts to be translated, such as product introductions, with the translation going on, more and more translation memories will be accumulated, and with the help of the AI translation, such as Xiaoniu translation which is capable of utilizing translation memories and term banks uploaded by the user, the human-machine interactive translation model will greatly promote the efficiency and accuracy of translation projects.

Through comparative analysis of translation results from various AI translations such as Google translation, Bing translation, Baidu translation, Sogou translation, Youdao translation and Xiaoniu translation, it is found that these AI translation systems produce translations that are complementary to each other as each has its own advantages and disadvantages. Therefore, for more efficient usage of AI translation in the human-machine interactive model, there is a need to employ several AI translation systems at the same time for reference and complementarity. In this regard, the Snowman CAT is a good choice as it provides five machine translation systems at the same time for comparison. In contrast, other CAT tools usually use only one machine translation system at a time.

\section{QUESTIONS FOR CLARIFICATION}

With regard to the usage of AI translation, there are some questions that need to be clarified.

Is the employment of the human-machine interaction translation model much more troublesome than direct human translation? Is the quality of machine translation not so reliable that it is more advisable to do human translation? These may be questions that translation teachers, students, and even researchers, will have.

It is a fact that in face of the huge amount of translation in the big data age, people in the language service industry believe that human assisted machine translation (HMT) is the trend of the future. It should not be ignored by translation teachers, students and researchers.

According to a survey of 50 junior students of a ChineseEnglish translation course, $100 \%$ of the students will use AI translation in the process of translation. What's more, some of them admit that machine translation sometimes is better than theirs. Thus, instead of turning a blind eye to this phenomenon, we'd better face up to it (indeed, more and more translators cooperate with AI translation) so as to make use of the best that AI translation can offer. And, before it goes out of control in 
regard to students' use of AI translation, teaching of postediting to students is urgently needed.

\section{IMPLICATIONS}

\section{A. The Role of AI Translation in Literary Translation}

AI translation is of high quality for some text types, such as news. However, it is generally agreed that AI translation is not up to literary translation. For example, Hu Kaibao believes that Machine translation is usually suitable for the translation of stylized or informative texts such as scientific and legal texts, while the translation of expressive texts such as novels, essays and poems needs to be undertaken by human translators [6]. We believe that although AI translation is not yet competent for literary translation, if the translator adopts the humanmachine interactive translation model, it is not without advantages and worth promoting. In the human-machine interaction model, the role of AI translation in literary translation is as follows:

(1) Some terms and sentences can be translated automatically and accurately, with translation memories and term banks uploaded to the AI translation server such as that of Xiaoniu translation. It is hoped that more AI translation services will provide users with the function of uploading translation memories and term banks to their server.

(2) It can be used for the training of literary translators in the new era of AI and big data. AI translation is rapidly evolving in terms of translation speed and quality, thanks to the increasingly growing corpus it has access to. With a data bank far larger than can be imagined, AI translation is now able to come up with translations that students majoring in translation can learn from. Moreover, AI translation is quite different from SMT in terms of error types in literary translation. If well studied and then utilized, the training of students' post-editing ability will be done more efficiently and fruitfully.

(3) It can be used for the Chinese-English translation of some types of literary works, such as Chinese web literature now popular at home and abroad. Novels of this kind are usually as long as millions of words, which makes it something unimaginable for human translators alone to put just some of them into English or to keep the translation in pace with the novel update. With the human-machine interactive translation model, however, it is possible and actually being done by Babel Novel, whose current AI machine can automatically monitor, translate and publish Chinese web literature novels without manual revision process, thus increasing the efficiency of the industry by 3600 times (for it takes AI translation only 1 second to render into English what it takes human translators 1 hour to finish), and reducing the cost to $1 / 100$ of the human translation.

\section{B. Reform of the Mode of Translation Talent Training and Translation Teaching}

AI translation has a subversive influence on translation process. Therefore, it is necessary to rethink the traditional training mode of translation talents and innovate translation teaching methods. Human-machine interaction is the preferred mode of translation. Correspondingly, both research and teaching should adapt in the following aspects.
Firstly, familiarizing translation teachers with translation technology. In China, most translation teachers need traning with regard to translation technology. In this regard, TAC, Translators Association of China, has done a lot by offering training courses to translation teachers, who will get certificates from TAC if they pass these courses.

Secondly, offering undergraduate students translation courses related to translation technology and AI translation. These courses, thought most of them are currently offered for MTI (Master of Translation and Interpreting) majors, should be offered at the undergraduate level, including corpus linguistics, computer-assisted translation, machine translation and postediting.

Thirdly, reforming translation teaching pedagogy. In the era of AI translation, the ways to reform and improve translation teaching include increasing the post-editing training of machine translation. It is a pity that most translation textbooks do not have any relevant chapter to deal with machine translation post-editing. Efforts are urgently needed in this regard. As for translation courses, more focus should be put on students' postediting competence cultivation, comparison between human translation and machine translation, contrastive translation analysis, acquisition of translation technology such as CAT tool usage, corpus construction and retrieval, online searching skills, and so on.

\section{Reform of Translation Tests}

Translation tests, such as Chinese-English translation in TEM- 8 and the Ministry of Personnel Translation Certificate Test, should allow the use of AI translation in addition to dictionaries. This can be done by means of a computer-based test, which is already available for tests such as TOEFL. This test method is closer to the actual translation operation. How to carry out computer-based translation tests needs to be studied.

\section{CONCLUSION}

AI translation should be treated with an objective attitude. Its so-called threat such as replacing human translation, should not be exaggerated, nor should AI translation be belittled. In the future, AI translation will continue to maintain a rapid development, and its translation quality will continue to improve. Facing the opportunities and challenges brought about by it, we should prepare for the rainy day and carry out relevant research on it. On the one hand, we should join hands with AI translation providers to promote the quality of AI translation, while on the other hand, we should promote the human-machine coupling, and enhance the efficiency and accuracy of human-assisted AI translation, which will be an important research field in the future.

As far as AI translation service is concerned, its evolution will also be a process of survival of the fittest, and some AI translation service providers may gradually lose their users in competition with other providers who are able to offer better quality translations. However, no matter how it develops, AI translation can not completely replace human translation, but the identity of the translator may change, and the role of the translator may change from translator alone to both translator and $\mathrm{AI}$ translation reviser, for whom post-editing competence 
is required, and the translator who lacks post-editing skills may gradually be less and less competitive.

In the future, human-machine interaction will become more and more popular, and the combination of the high efficiency of machine translation and the accuracy of human translation will achieve a leap in both quantity and quality. In this perspective, colleges and universities need to innovate translation teaching, train qualified personnel for AI translation and post-editing, and many problems need to be solved, such as the compilation of translation technology textbooks, the training of teachers with expertise in translation technology, the offering of translation technology courses, and so on. In addition, in translation studies more attention should be paid to the theory and practice of AI translation and its quality assessment, which will help to improve AI translation quality and open up a new era for the integration of translation studies and translation technology.

\section{REFERENCES}

[1] Zhang Xiaojun, He Ying. Translation's Technological Turn: A Report from the FIT 20th World Congress (In Chinese) [J]. Chinese Translators Journal, 2014(6):74-77.

[2] Diao Hong. Review of Translation Technology Studies in China (In Chinese) [J]. Journal of Beijing International Studies University, 2017(6):69-81, 125

[3] Wang Kefei. Exploring Corpus-based Translation Studies (In Chinese) [M]. Shanghai: Shanghai Jiaotong University Press, 2012.

[4] Cui Qiliang, Li Wen. Post-editing Error Type Research Based on English-Chinese Translation of Technical Texts (In Chinese) [J]. Chinese Science \& Technology Translators Journal, 2015(4):19-22.

[5] Wu, Y. et al. Google's Neural Machine Translation System: Bridging the Gap Between Human and Machine Translationy [J]. arXiv reprint arXiv:1609.08144, 2016.

[6] Hu Kaibao, Li Yi. Research on Features of Machine Translation and its Relations with Human Translation (In Chinese) [J]. Chinese Translators Journal, 2016(5):10-14. 